\title{
Turbulent transport in the TCV SOL
}

\author{
O. E. Garcia ${ }^{a, *}$, R. A. Pitts ${ }^{\text {b }}$, J. Horacek ${ }^{b}$, A. H. Nielsen ${ }^{\text {a }}$, \\ W. Fundamenski ${ }^{\text {c }}$, J. P. Graves ${ }^{\text {b }}$, V. Naulin ${ }^{\text {a }}$, J. Juul Rasmussen ${ }^{\text {a }}$ \\ a Association EURATOM-Ris $\emptyset$ National Laboratory, OPL-128 Ris $\phi$, DK-4000 Roskilde, Denmark \\ ${ }^{\mathrm{b}}$ Association EURATOM-Confédération Suisse, CRPP, EPFL, CH-1015 Lausanne, Switzerland \\ ${ }^{\mathrm{c}}$ EURATOM/UKAEA Fusion Association, Culham Science Centre, Abingdon, OX14 3DB, UK
}

\begin{abstract}
From probe measurements in the SOL of TCV it is demonstrated that while the particle density profile becomes increasingly broader with increasing line averaged density, the radial variation of the fluctuation statistics remains the same. Excellent agreement is found between the experimental measurements and an interchange turbulence simulation, revealing intermittent plasma transport dominated by radial motion of plasma filaments. On the basis of this favourable code-experiment agreement, it is concluded that no reliable parameterization exists for the turbulent particle flux in terms of effective diffusion and convection coefficients.
\end{abstract}

Corresponding author address: Ris $\varnothing$ National Laboratory,

OPL-128, PO Box 49,

DK-4000 Roskilde,

Denmark

Corresponding author email: odd.erik.garcia@risoe.dk

Presenting author: Richard Pitts

Presenting author email: richard.pitts@epfl.ch 


\section{INTRODUCTION}

Turbulent transport in the scrape-off layer (SOL) of magnetically confined plasmas is routinely quantified in terms of effective diffusion coefficients. This has usually been justified on the basis of the so-called flux-gradient paradigm, namely that turbulence and hence anomalous transport is driven by gradients in the local plasma parameters. This paradigm underlies most theories of collective motions and transport in the SOL [1].

However, a number of experimental investigations have shown that turbulent transport in the SOL is dominated by radial motion of field-aligned plasma filaments, observed as blobs in the plane perpendicular to the magnetic field. These structures seem to be formed in the vicinity of the last closed magnetic flux surface and propagate radially far into the SOL. Such intermittent transport has been found to prevail in virtually all confinement regimes and may significantly influence plasma interaction with main chamber walls in future long pulse devices [2-11].

It has been recently demonstrated that two-dimensional interchange turbulence simulations are able to reproduce the radial variation of fluctuation statistics measured in the SOL of TCV. The simulations clearly indicate that turbulence is driven in the steep profile region inside the SOL, from where plasma blobs intermittently erupt into the open field line region. This is likely to be the cause of the experimentally observed broad particle density profiles, large relative fluctuation levels, and the skewed conditional waveforms and single-point probability distributions found in the SOL plasma [11-13].

In this contribution we first analyze a set of density ramp discharges on TCV and compare probe measurements with the result of a turbulence simulation. We then address the question of whether the turbulent particle transport can be parameterized in terms of effective diffusion and convection coefficients, as is routinely estimated from experimental measurements [5-8] and assumed in analytical and transport code modelling of SOL plasmas [14-17].

\section{SOL PROFILES AND FLUCTUATIONS}

For this investigation we analyze probe data from a set of identical, deuterium fuelled, ohmically heated, density ramp pulses with plasma current 340kA. A fast reciprocating Langmuir probe array measures the ion saturation current and floating potential poloidally halfway between the outer midplane and the X-point in these lower single null discharges. Two probe reciprocations 
are performed for each discharge, corresponding to line averaged densities, $\bar{n}_{\mathrm{e}}$, of approximately $4.5 \times 10^{19} \mathrm{~m}^{-3}$ and $11 \times 10^{19} \mathrm{~m}^{-3}$, respectively. These low and high density cases will henceforth be denoted respectively by RCP1 and RCP2. Further details of the experiment and measurements can be found in Refs. [9-11]. In particular, Ref. [11] describes the model equations, geometry and results of interchange turbulence simulations that have been performed for the outboard TCV SOL and with which we present further comparison here against experimental measurements.

Figure 1 presents the time-averaged particle density profiles for the two probe reciprocations. The normalized radial coordinate, $\rho$, is defined as zero at the separatrix and unity at the wall radius. In common with observations on several other tokamaks, the profile in the main SOL becomes broader with increasing $\bar{n}_{\mathrm{e}}$ [4-8]. Assuming exponentially varying profiles results in the particle density scale lengths, defined by

$$
\lambda_{n}=-\frac{n}{\partial n / \partial r}=-\frac{1}{\partial \ln n / \partial r}
$$

presented in Fig. 2. In the low-density case the profile is steep in the vicinity of the separatrix with a scale length of about $0.5 \mathrm{~cm}$. Further out the profile is much broader, with an estimated scale length of $2.3 \mathrm{~cm}$. In the high density case the profile is approximately exponential over the central part of the main SOL with a scale length of $4.5 \mathrm{~cm}$. An exponential fit from the separatrix position to the wall radius yields a scale length of $3.8 \mathrm{~cm}$ [11]. This broadening of the plasma profile, in both scale length and radial extent, leads to enhanced levels of plasma interaction with the main chamber wall and is generally attributed to turbulent transport. The latter is supported by results from an edge-SOL electrostatic (ESEL) interchange turbulence simulation for which the model parameters are adapted to the high density TCV case appropriate to RCP2 [11]. The result from this simulation has been included along with the experimental data in Fig. 1 and in many of the subsequent figures presented here.

Despite significant differences in the time-averaged profile with increasing $\bar{n}_{\mathrm{e}}$, the fluctuation statistics have a similar radial variation over the SOL region where the profile is broad. This is clearly seen from the radial variation of the standard deviation, skewness and flatness of the particle density fluctuations, shown in Figs. 3-5. The relative fluctuation level is of order unity and the abundance of positive bursts in the probe time series leads to statistical distributions which are strongly skewed and flattened. As seen in the figures, these statistical moments are well reproduced by the turbulence simulation for both the high and low density cases of RCP1 and RCP2. This is in fact a manifestation on a small scale (namely, for just two different densities in the same discharge) 
of the universal statistical properties seen in the TCV probe time series across a wide range of discharge parameters $[9,10]$. The good agreement between code and experiment demonstrates that the high relative fluctuation levels and resulting broad plasma profiles are due to radial interchange motions of plasma filaments [11-13]. This is therefore also very likely to be what underlies the radial increase in the statistical moments seen in these experiments. Based on this insight, it is not at all evident that the radial turbulent particle flux density, $\Gamma$, shown in Fig. 6 , can be parameterized in terms of an effective turbulent diffusion or convective velocity.

\section{TURBULENT TRANSPORT}

Transport of a passive scalar field subject to advection by a random, small-scale velocity field can be described on large scales by an effective diffusivity provided the turbulent flow possesses certain symmetry properties [18-20]. A separation of the spatial and temporal scales between the turbulent motions and the passive scalar field is inherent in the derivation and hence for the concept of an effective diffusivity. Based on this theoretical foundation, turbulent transport in magnetized plasmas is often described in terms of an effective diffusion coefficient for the particle transport defined by

$$
\Gamma=-D_{\text {eff }} \frac{\partial n}{\partial r}=\frac{n D_{\text {eff }}}{\lambda_{n}}
$$

Using the separatrix values $n \approx 2 \times 10^{19} \mathrm{~m}^{-3}, \Gamma \approx 3 \times 10^{21} \mathrm{~m}^{-2} \mathrm{~s}^{-1}$ and $\lambda_{n} \approx 4 \mathrm{~cm}$ for the high density TCV case, the effective diffusivity is $6 \mathrm{~m}^{2} \mathrm{~s}^{-1}$. The radial variation of $D_{\text {eff }}$ for the two density cases and the turbulence simulation is presented in Fig. 7. In the low density case, $D_{\text {eff }}$ increases with radius, while at high density the effective diffusivity has a broad peak in the middle of the SOL. Thus, the turbulent particle flux cannot be unambiguously parameterized in terms of an effective diffusivity. We also note that $D_{\text {eff }}$ is an order of magnitude larger than the Bohm diffusion level, $D_{\mathrm{Bohm}}=T_{\mathrm{e}} / 16 e \mathrm{~B}$, which is approximately $1 \mathrm{~m}^{2} \mathrm{~s}^{-1}$ for the separatrix parameters chosen above.

The failure of the diffusion ansatz as a description of experimental measurements, together with the increasing experimental evidence for intermittent SOL transport caused by radial motion of plasma filaments, have motivated the heuristic application of an effective convective velocity, $V_{\text {eff }}$, for the parameterization of the turbulent particle flux density [6-8, 15-17],

$$
\Gamma=n V_{\text {eff }}
$$


For the same separatrix plasma and flux density values used above, the convective velocity defined by Eq. (3) is $150 \mathrm{~m} \mathrm{~s}^{-1}$. Figure 8 shows that $V_{\text {eff }}$ varies significantly over the radial extent of the SOL at low density (RCP1). At high density (RCP2), the effective convective velocity is roughly constant over the main SOL. It is interesting to note that in the region with broad particle density profiles, $V_{\text {eff }}$ is roughly the same for the two density cases. However, neither the diffusion nor the convection ansatz provides a unified description of the various plasma states.

In analytical and transport code modelling of SOL plasmas, the transport is often described as a simple linear combination of effective diffusion and convection [15-17],

$$
\Gamma=-\widehat{D}_{\text {eff }} \frac{\partial n}{\partial r}+n \widehat{V}_{\text {eff }}
$$

Of course, these transport coefficients cannot be inferred from the above estimates which describe the transport as being due to either diffusion or convection. We have thus used hat symbols to indicate that the coefficients in Eq. (4) are different from those defined by Eqs. (2) and (3). From Eq. (4) it follows that the ratio of the particle flux and number density can be written as

$$
\frac{\Gamma}{n}=\widehat{V}_{\text {eff }}-\frac{\widehat{D}_{\text {eff }}}{n} \frac{\partial n}{\partial r}=\widehat{V}_{\text {eff }}+\frac{\widehat{D}_{\text {eff }}}{\lambda_{n}} .
$$

Thus, if the turbulent particle flux can be parameterized in terms of a radially constant effective diffusivity and a convective velocity, a plot of $\Gamma / n$ versus $1 / \lambda_{n}$ would be a straight line from which the transport coefficients could be trivially determined [21]. Specifically, $\widehat{V}_{\text {eff }}$ would be given by

the point where the approximately linear curve intersects the vertical axis and $\widehat{D}_{\text {eff }}$ would be given by the slope of the curve. As can be seen from Fig. 9, this is not at all the case for the experimental measurements considered here. This clearly indicates that, at least for these TCV plasmas, no reliable parameterization of the radial turbulent transport exists if this transport is to be described purely in terms of an effective particle diffusivity and a convective velocity.

\section{CONCLUSIONS}

Analysis of probe measurements in a set of TCV density ramp experiments has shown that the particle density fluctuations in the SOL exhibit universal properties despite significant changes in the average profiles [9]. The relative fluctuation level, skewness and flatness are all increasing functions of radius. Two-dimensional interchange turbulence simulations adapted to the experiments are in quantitative agreement for the radial variation of the profiles and fluctuation statistics 
[11]. It is thus clear that the turbulent transport in the SOL region is highly intermittent and dominated by radial motion of plasma filaments. These structures display a complex spatio-temporal evolution, including acceleration and dispersion processes [13].

Turbulent transport in the SOL is routinely described in terms of effective diffusion and convection coefficients. Here we have questioned the physical foundation for this common practice. It is found that for the TCV SOL, neither the diffusion nor the convection ansatz are able to uniformly describe the experimental measurements. This is hardly a surprising result, bearing in mind that SOL plasmas are generally characterized by fluctuation levels of order unity, absence of scale separation between profiles and fluctuations, and most likely anisotropic and inhomogeneous turbulent velocity fields. This negative result does of course not undermine the usefulness of analytical and transport code modelling based on effective diffusion and convection coefficients. It does, however, provide strong motivation for further investigations of SOL transport based on first principles physics.

\section{Acknowledgments}

O. E. G. was supported with financial subvention from the Research Council of Norway and the Danish Research Agency. 
[1] J. W. Connor et al., Nucl. Fusion 39 (1999) 169.

[2] J. A. Boedo et al., Phys. Plasmas 10 (2003) 1670.

[3] S. J. Zweben et al., Nucl. Fusion 44 (2004) 134.

[4] D. L. Rudakov et al., Plasma Phys. Control. Fusion 45 (2005) 1589.

[5] B. LaBombard et al., Phys. Plasmas 8 (2001) 2107.

[6] B. Lipschultz et al., Plasma Phys. Control. Fusion 44 (2002) 733.

[7] B. Lipschultz et al., Plasma Phys. Control. Fusion 47 (2005) 1559.

[8] D. G. Whyte et al., Plasma Phys. Control. Fusion 47 (2005) 1579.

[9] J. P. Graves et al., Plasma Phys. Control. Fusion 47 (2005) L1.

[10] J. Horacek et al., Czech. J. Phys. 55 (2005) 271.

[11] O. E. Garcia et al., Plasma Phys. Control. Fusion 48 (2006) L1.

[12] O. E. Garcia et al., Phys. Rev. Lett. 92 (2004) 165003; Phys. Plasmas 12 (2005) 062309; Phys. Scr. T122 (2006) 89.

[13] O. E. Garcia et al., Phys. Plasmas 12 (2005) 090701.

[14] ITER Physics Basis, Nucl. Fusion 39 (1999) 2391.

[15] A. Yu Pigarov et al., Phys. Plasmas 9 (2002) 1287.

[16] P. C. Stangeby, Phys. Plasmas 9 (2002) 3489.

[17] R. Schneider et al., Contrib. Plasma Phys. 46 (2006) 3.

[18] A. J. Majda and P. R. Kramer, Phys. Rep. 314 (1999) 1.

[19] N. H. Bian and O. E. Garcia, Phys. Plasmas 12 (2005) 042307.

[20] H. K. Moffatt, Rep. Prog. Phys. 46 (1983) 621.

[21] V. Naulin, Phys. Rev. E 71 (2005) 015402. 


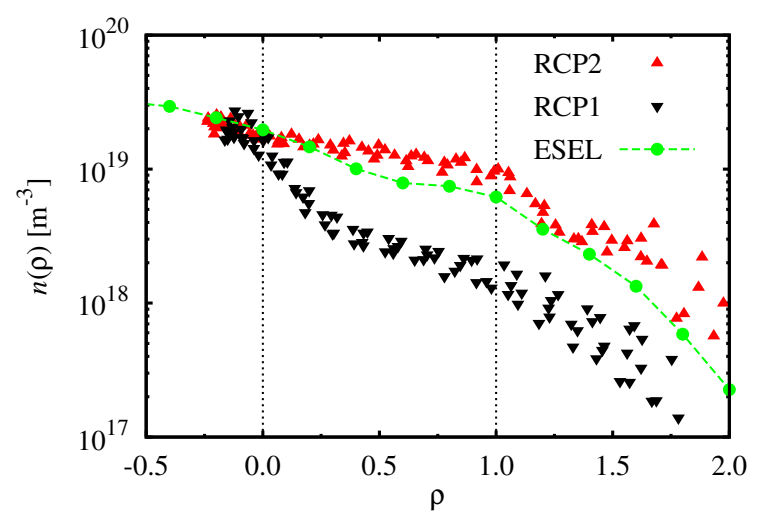

FIG. 1: Time-averaged particle density profiles.

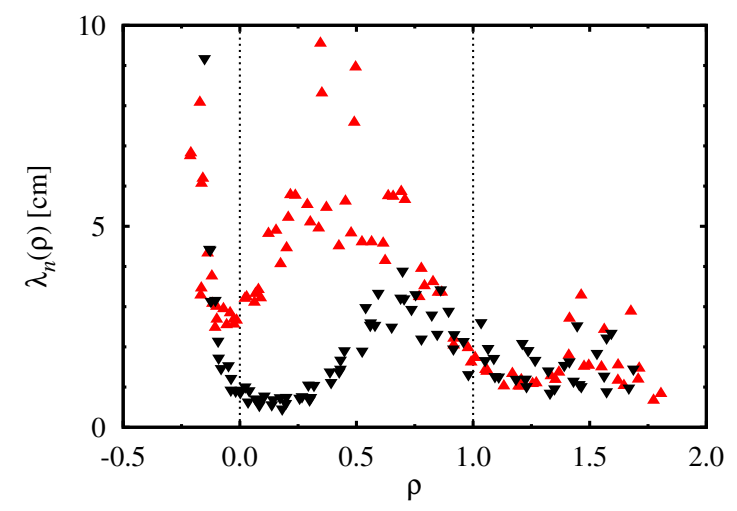

FIG. 2: Length scale of the particle density profiles.

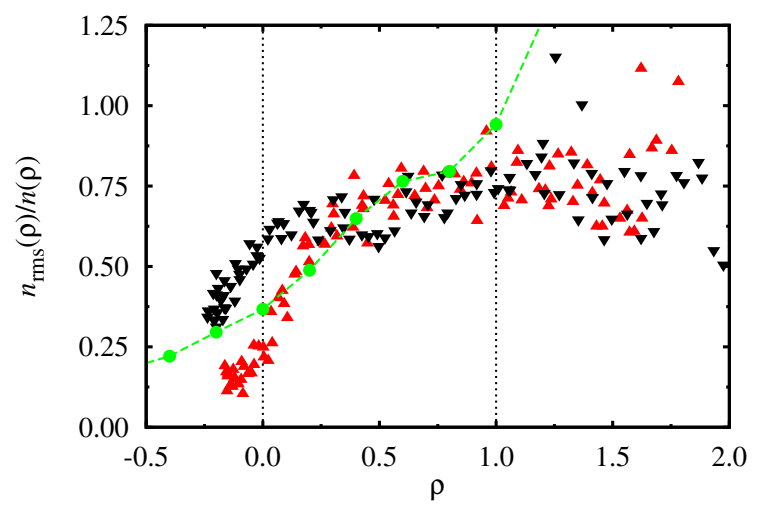

FIG. 3: Relative particle density fluctuation levels. 


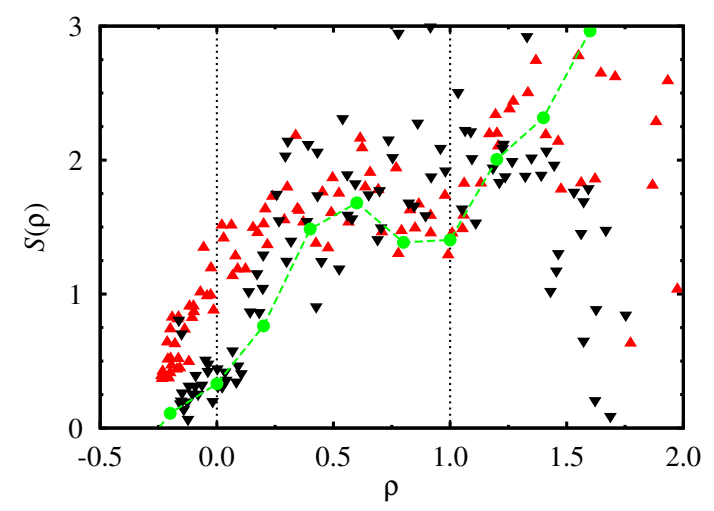

FIG. 4: Skewness of the particle density fluctuations.

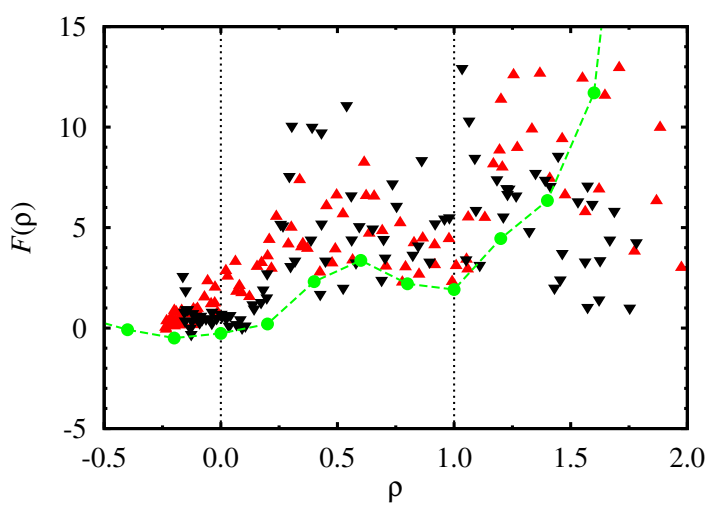

FIG. 5: Flatness of the particle density fluctuations.

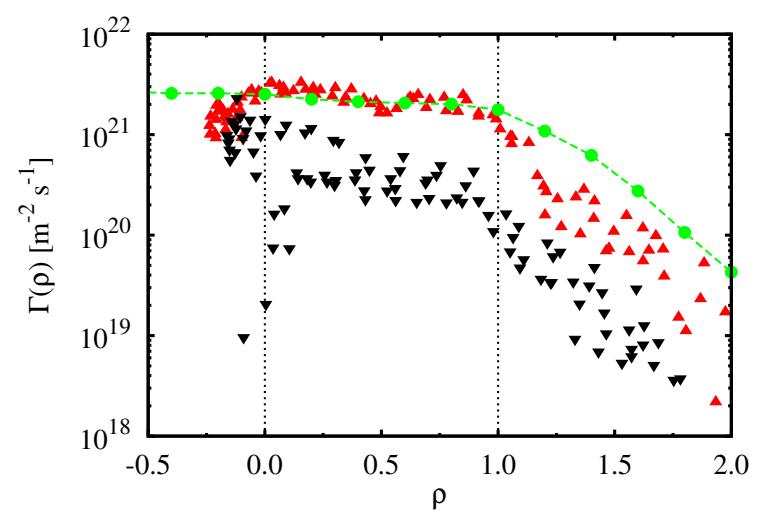

FIG. 6: Time-averaged profile of the radial turbulent particle flux. 


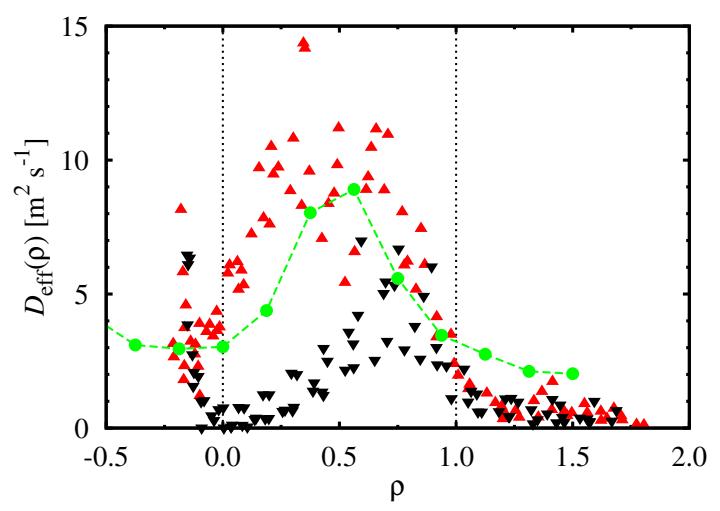

FIG. 7: Effective diffusion coefficient defined by $\lambda_{n} \Gamma / n$.

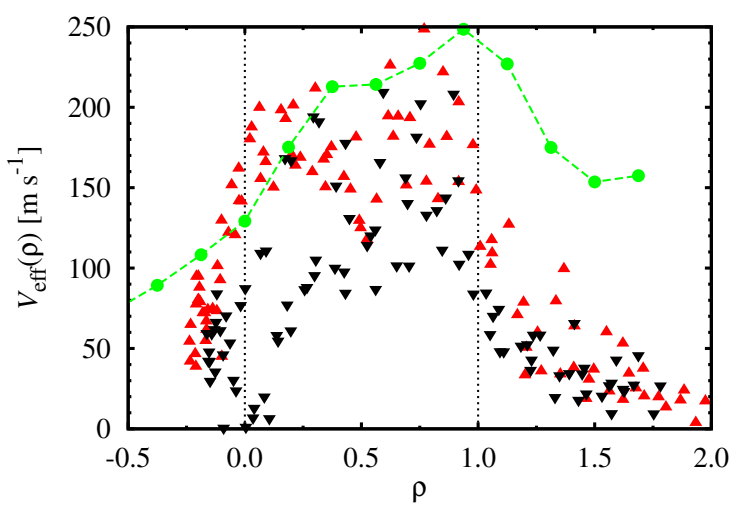

FIG. 8: Effective convective velocity defined by $\Gamma / n$.

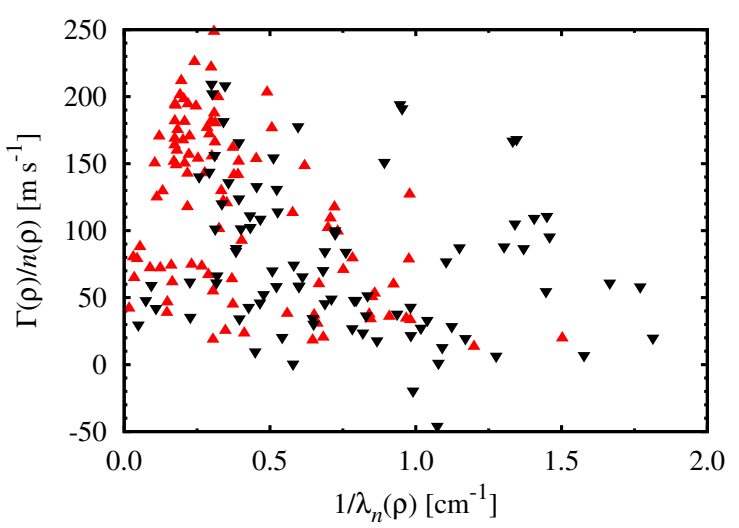

FIG. 9: Scatter plot for the flux-gradient relation. 